\title{
A Tangible Programming Language for the Educational Robot Thymio
}

\author{
Andrea Mussati \\ Mobots group of the Biorobotics \\ Laboratory \\ Ecole Polytechnique Fédérale de \\ Lausanne (EPFL) \\ Lausanne, Switzerland \\ andrea.mussati@epfl.ch
}

\author{
Christian Giang \\ Mobots group of the Biorobotics \\ Laboratory \\ Ecole Polytechnique Fédérale de \\ Lausanne (EPFL) \\ Lausanne, Switzerland \\ christian.giang@epfl.ch \\ Francesco Mondada \\ Mobots group of the Biorobotics \\ Laboratory \\ Ecole Polytechnique Fédérale de \\ Lausanne (EPFL) \\ Lausanne, Switzerland \\ francesco.mondada@epfl.ch
}

\author{
Alberto Piatti \\ Department of Education and Learning \\ (DFA) \\ University of Applied Sciences and Arts \\ of Southern Switzerland (SUPSI) \\ Locarno, Switzerland \\ alberto.piatti@supsi.ch
}

\begin{abstract}
In the past, the use of tangible programming languages has shown several advantages compared to screenbased graphical programming languages. Especially when presented to novices, such interfaces may represent a more intuitive and straightforward alternative to teach basic computer science and programming concepts. Previous studies have reported increased interest and improved collaboration when tangible programming languages were used. However, additional financial expenses have often hindered the use of such interfaces in formal education settings. This work therefore presents a low-cost and customizable solution of a tangible programming language for Thymio, an educational robot widely used in primary and secondary schools. Using a computer vision algorithm, graphical icons printed on paper are captured by a camera, and subsequently interpreted and sent to the robot for execution. Two user studies with in total 77 university students showed promising results, indicating that the devised interface can elicit more interest and a higher level of collaboration within groups.
\end{abstract}

Keywords-Educational robotics, personalized learning, tangible programming,

\section{INTRODUCTION}

Previous studies have shown that introducing robots as learning artifacts in classrooms can be beneficial in many ways. Not only can it increase students' general interest in STEM (science, technology, engineering and mathematics) disciplines [1], but it can also foster their communication skills and team work [2]. One main objective of introducing educational robots into classrooms is to teach basic computer science and programming concepts. In this context, the use of tangible programming languages (TPLs) has suggested several advantages and many attempts have been made to develop such interfaces [3], [4]. TPLs usually consist of physical blocks which can be assembled to program either physical objects (e.g. robots) or virtual agents (e.g. digital avatars). Previous work has demonstrated that compared to screen-based graphical programming languages, TPLs may improve collaboration within groups [5], increase interest in the learning topics themselves [6], and lead to better learning

This work has been partially supported by the Swiss National Science Foundation through the National Centre of Competence in Research Robotics. gains [7]. The benefits of providing all group members a way to interact with the interface simultaneously, has been discussed before [8] and can be considered a main advantage of TPLs. Moreover, by involving tangible objects, TPLs may better exploit the socio-constructivist roots of educational robotics and provide opportunities to implement more playful ways of learning, resulting in activities previously referred to as "educational augmented tabletop games" [9]. Despite these affordances, the use of TPLs for educational robots in formal education settings is still relatively sparse. Especially considering the high costs for educational robotics materials (i.e., the expenses for robots, computers and/or tablets), the additional financial efforts for TPLs have represented a crucial argument against their use in schools. Aiming at addressing this issue, the goal of this work is to develop a low-cost and customizable solution of a TPL for Thymio, an educational robot, increasingly being used in primary and secondary schools in Switzerland and other European countries to introduce students to science and technology [10].

\section{DEVELOPMENT OF THYMIO TPL}

To date, the most frequently used programming language for the Thymio robot is the graphical interface VPL [11]. It is a block-based programming language, where two types of blocks, events and actions, are combined to create programs using a drag-and-drop interface (Fig. 1, left). Orange event blocks depict different kinds of inputs the robot can receive (e.g., pressed buttons, infrared sensor data, acoustic signals), while the blue action blocks depict the actions that the robot can perform (e.g., displacements, color of LEDs, playing sounds). Some blocks are parametric, allowing for instance to select the different infrared sensors of the robot. Multiple lines of event-action combinations can be merged to create complex behaviors of the robot. In contrast to sequential programming languages, the order of the lines in this event-based programming language does not matter.

For the development of the tangible programming language Thymio TPL, one of the design goals was to allow learners a smooth transition between the two interfaces TPL and VPL. Therefore, the same blocks and logical structure were maintained (Fig. 1, right). Minor modifications were applied to some parametric presentations (cubes instead of sliders) to facilitate the implementation. Moreover, aiming at

(c) 2019 IEEE. Personal use of this material is permitted. Permission from IEEE must be obtained for all other uses, in any current or future media, including reprinting/republishing this material for advertising or promotional purposes, creating new collective works, for resale or redistribution to servers or lists, or reuse of any copyrighted component of this work in other works. 


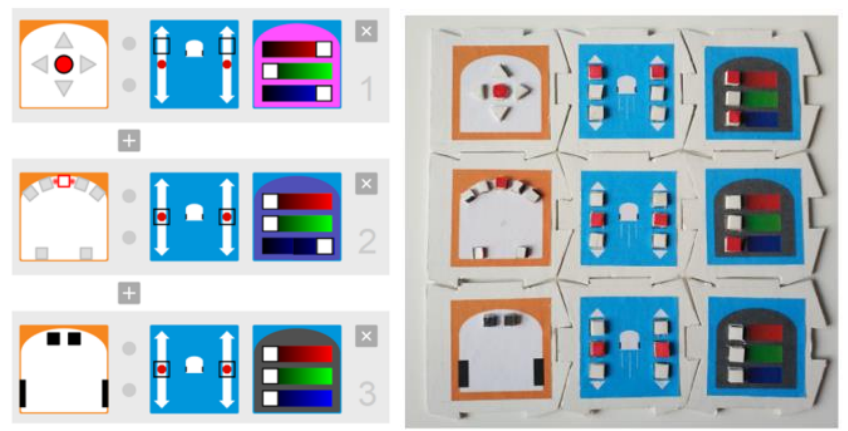

Fig. 1. Example of the same program implemented in Thymio VPL (left) and Thymio TPL (right). When the central button is pressed, Thymio moves forward and lights up in purple. When the central front sensor detects an obstacle, Thymio stops and lights up in blue. When the bottom sensors detect void (e.g. at the edge of a table), Thymio stops and turns off the lights.

a low-cost solution that could be easily reproduced by teachers and students, simple craft materials, that can be usually found in most classrooms, were used for the fabrication of the blocks. The symbols were printed on paper and glued on cardboard blocks, that could be assembled in a puzzle-like fashion to form the program instructions. Parametric capabilities were given by small cubes of different colors that could be embedded in slots carved into the cardboard. The assembled sets of instructions were then captured by an external webcam, allowing for a complete separation of the computer from the workspace. The image data was automatically processed by a computer vision algorithm and the instructions sent to the robot for execution through a wireless connection.

\section{USER STUDIES}

\section{A. First user study}

A first user study with 38 university students was organized to assess the educational potential of Thymio TPL. None of the students have worked with any of the interfaces before and each student gave their informed consent to participate in the study. Students worked in eight groups of 45 students and each group tested both interfaces. After an introductory speech, each group was given a set of Thymio programming tasks (e.g., "Make Thymio follow a black line"), and had six minutes to complete them. The order of presentation for both interfaces was randomized: while half of the groups started with VPL, the other half started with TPL. For each interface, the groups worked in a separate workspace on opposite sides of the room (Fig. 2). At the end of the six minutes the groups switched platforms and were given a new set of tasks to perform in another six minutes. The number of people participating in the work (i.e., those who, during the six minutes work, were actively participating in solving the tasks at hand) was assessed by direct observations of two experimenters independently taking written notes during the tests. Subsequently, the results were discussed among both experimenters to reduce observational biases. The results of this study are presented in Section IV.

\section{B. Second user study}

Based on the results of the first study, a second study was organized with another 39 university students, using a modified protocol. The introduction speech and task sheets were changed, accounting for the experience gathered from the previous study. The objective of this second study was to gather more quantitative data, providing insight into how the

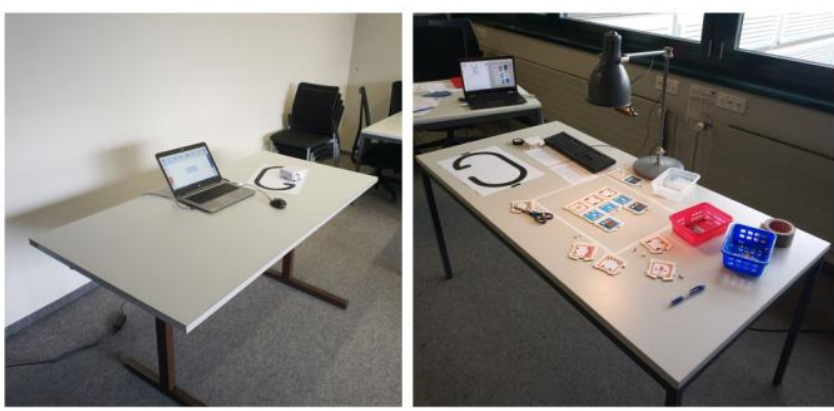

Fig. 2. Workspaces for groups working with Thymio VPL (left) and Thymio TPL (right) during the user studies.

students interacted and collaborated while using each interface. Therefore, each group was videotaped while working with each interface. The video recordings were then analyzed by two experimenters independently and the results subsequently discussed among both experimenters to reduce observational biases. Each student gave their informed consent to participate in the study and to being video recorded during the study. For this study, the timing and the type of the students' interactions with the platform and within the group were analyzed in detail. Therefore, four levels of interaction with the platforms were established. Every student was classified to be in one of these four states at any point during the six minutes:

1. Not Interacting, Not Interested (NINI): e.g. checking the mobile phone, looking at other parts of the room.

2. Not Interacting, Interested (NII): not working on the platform nor discussing with the others, but watching the platform and the work being performed on it.

3. Non-Direct Interaction (NDI): discussing strategies, proposing solutions, telling other people to perform actions on the platform.

4. Direct Interaction (DI): directly interacting with either the programming platform or with the robot to test the program.

Similarly, three states of collaboration among the members of a group were established. Every student was classified to be in one of these three states at any point during the six minutes, independently and in parallel with their classification for interaction:

1. Not Collaborating (NC): either not working on the tasks or working independently of the others.

2. Verbal Collaboration (VC): collaborating with others by discussing strategies, or by telling other people what action to perform.

3. Physical Collaboration (PhC): directly working in parallel and with coordinated action on different parts of the system, e.g. one person programming while another tests the result on the robot.

If students were both verbally and physically collaborating, they were classified as $\mathrm{PhC}$. The amount of time spent in each state of both dimensions was analyzed for each student independently. Finally, the results for both platforms were compared against each other. Moreover, qualitative data was collected by video analysis: whether one or a few persons would be the only ones directly interacting with the platform; how uniform the experience would be between participants,

(C) 2019 IEEE. Personal use of this material is permitted. Permission from IEEE must be obtained for all other uses, in any current or future media, including reprinting/republishing this material for advertising or promotional purposes, creating new collective works, for resale or redistribution to servers or lists, or reuse of any copyrighted component of this work in other works. 
i.e., how similar the activity profile of each student would be; and whether the group would continue working on the tasks after being told that the time was over.

\section{RESULTS}

\section{A. First user study}

Table I presents a summary of active participation in solving the presented tasks depending on the platform for the first user study. Students were classified as not participating and actively participating based on the direct observation of the experimenters. While two thirds (25) of the students appeared to be rather passive when working with the graphical programming language VPL, this was true for only one third (13) of the students who worked with Thymio TPL. For active participation instead, reverse conditions were found (13 students actively participating in VPL against 25 students in TPL). Based on the results of this study, a second user study was designed and conducted.

TABLE I

ACTIVE PARTICIPATION IN FIRST USER STUDY $(\mathrm{N}=38)$

\begin{tabular}{ccc}
\hline \hline Platform & Actively participating & Not participating \\
\hline Thymio $V P L$ & 13 & 25 \\
Thymio $T P L$ & 25 & 13 \\
\hline \hline
\end{tabular}

\section{B. Second user study}

In the second user study students' interaction and collaboration was analyzed based on video recordings made during the experiments. On average, the students working with VPL spent the majority of their time (170 seconds, corresponding to $47 \%$ of the six minutes) in the Non-Direct Interaction (NDI) state, acting on the platform only through others (Fig. 3). Almost a third of the time (30\%) was spent in a Non-Interacting state (NINI: $5 \%$; NII: $25 \%$ ), while only about one quarter of the time $(23 \%)$ was spent in direct interaction with either the VPL platform or the robot. In contrast, when working with TPL, the students spent the majority of their time in Direct Interaction with the tiles or the robot (DI: 44\%). A little less time was spent in Non-Direct Interaction (NDI: 39\%) and only about a sixth of the time in a Non-Interacting state (NINI: 2\%; NII: 15\%). A Wilcoxon signed-rank test validated a statistically significant difference $(p<0.01)$ for direct interaction between both platforms. As for collaboration, on average the students working with VPL spent most of their time (245 seconds, corresponding to $68 \%$ of the six minutes) in Verbal Collaboration (VC) with each other (Fig. 4), and more than a quarter of the time in NonCollaboration (NC, 26\%); mostly due to not participating in the activity at all (being in a Non-Interacting state), rarely because they were working on their own (e.g. silently checking the Thymio robot while the others were working on the program). Very little time $(6 \%)$ was spent in Physical Collaboration $(\mathrm{PhC})$; which mostly happened when one was testing the program on the Thymio robot while another corrected the code in VPL. In TPL, the majority of the time (45\%) was still spent in Verbal Collaboration (VC), however, the amount of time in this state was considerably lower than observed for VPL. Almost as much time (39\%) was spent in Physical Collaboration (PhC), mostly with students preparing blocks or building instructions in parallel, by often implicitly, sometimes explicitly, splitting the assembling task between individuals or subgroups. Finally, only about a sixth of the time was spent in Non-Collaboration (NC: 16\%), almost exclusively from students who were in a Non-Interacting

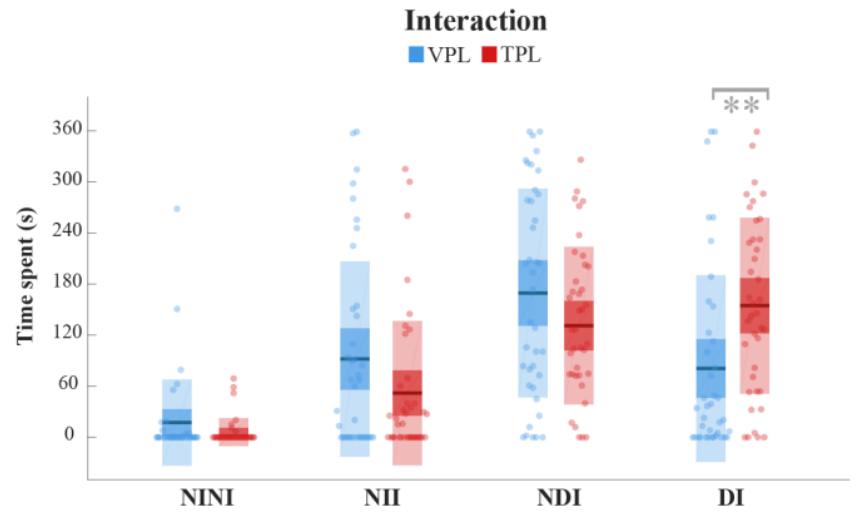

Fig. 3. Time spent in each interaction state for both interfaces in the second user study $(n=39)$. Colored dots represent the time spent in each state by each individual student. Colored lines indicate median values for each state. Dark areas indicate the $95 \%$ confidence intervals, bright areas indicate one standard deviation. Results of a Wilcoxon signed-rank test between values are indicated by asterisks $(* *, \mathrm{p}<0.01)$. NINI $=$ Not Interacting, Not Interested; NII = Not Interacting, Interested; NDI = Non-Direct Interaction; DI $=$ Direct Interaction.

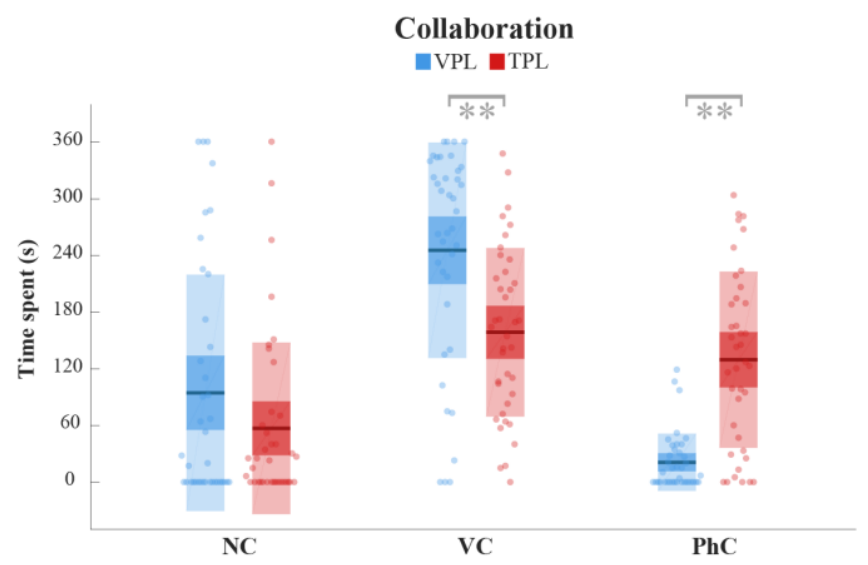

Fig. 4. Time spent in each collaboration state for both interfaces in the second user study $(n=39)$. Colored dots represent the time spent in each state by each individual student. Colored lines indicate median values for each state. Dark areas indicate the $95 \%$ confidence intervals, bright areas indicate one standard deviation. Results of a Wilcoxon signed-rank test between values are indicated by asterisks $(* *, \mathrm{p}<0.01)$. $\mathrm{NC}=$ Not Collaborating; VC $=$ Verbal Collaboration; $\mathrm{PhC}=$ Physical Collaboration.

state. A Wilcoxon signed-rank test validated statistically significant differences in Verbal Collaboration $(\mathrm{p}<0.01)$ and Direct Collaboration $(\mathrm{p}<0.01)$ between both platforms, indicating a shift of observed collaboration states from one platform to the other.

\section{Qualitative results}

From the video recordings made during the second user study, further qualitative analyses were performed: over the course of the six minutes of the VPL experiments, the number of interacting, interested, and collaborating students tended to peak in the first three minutes; while in the last three minutes those who were not interacting with the group tended to lose interest in the activity. On the opposite, interest appeared to be more constant during the TPL experiments; some students who weren't interacting with others continued manipulating tiles, even without major communication with the group. Moreover, those who at first were not collaborating in the activity tended to join in after the third or fourth minute. During the VPL experiments, most groups had only one to two students interacting with the platform; and usually another manipulating the Thymio robot; others would only help verbally. In contrast, on TPL, most groups had either four or

(C) 2019 IEEE. Personal use of this material is permitted. Permission from IEEE must be obtained for all other uses, in any current or future media, including reprinting/republishing this material for advertising or promotional purposes, creating new collective works, for resale or redistribution to servers or lists, or reuse of any copyrighted component of this work in other works. 
five students interacting with the tiles; and usually three to four of them would also manipulate the robot. Interestingly, even in strongly collaborating groups, when working with VPL, the main conversation would usually leave some people out. In TPL instead, those who would be left out of the conversation would either join in through interaction with the tiles (e.g. picking up a tile and pointing out its features) or gather in secondary groups building an instruction, that could eventually be added to the program being built, giving them an opportunity to rejoin the main conversation. Six minutes after the start of the experiment, each group was told that the time was up, and they were asked to switch platforms or head to the debriefing, respectively. Of the eight groups finishing the VPL experiments, three continued their work; two for less than two minutes, one for another five minutes. Of the eight groups finishing the TPL experiments, seven continued their work; five for less than two minutes; one for more than four minutes; another for more than eight.

\section{DISCUSSION}

Although the results presented in this work appear to be promising, they should rather be interpreted as preliminary findings on the educational potential of the devised platform. It should be acknowledged that the results presented in this study are mostly based on qualitative observations of the experimenters. Although all results were discussed and double-checked by both experimenters independently to reduce any kind of observational bias, this condition certainly represents a limitation of this study. Moreover, it can be assumed that representing interaction and collaboration using a set of predefined states may not exhaustively cover the complexity of both concepts. Likewise, the comparatively short testing time does not allow for generalization to regular educational robotics activities, which usually take longer. Nevertheless, the findings of this study illustrate the potentials of the devised Thymio TPL platform to foster active participation and collaboration within work groups. However, in order to draw more substantial conclusions, further studies with larger sample sizes from the actual target population, i.e., primary and secondary school students, have to be conducted, focusing on the learning gains achieved by both approaches. Future works should also focus on the usability of the platform, especially with regard to the use in classrooms. Thanks to the simple craft materials used for the fabrication of the TPL blocks, they can be easily reproduced by teachers and students in a low-cost manner. Indeed, in the simplest implementation, the TPL blocks can be purely paper-based, and the computer vision algorithm can be executed on a tablet or smartphone with an integrated camera. This provides schools, which are already using the Thymio robot, a possibility to acquire the platform without any additional expenses. With regard to accessibility, this represents a major advantage of the platform, since Thymio robots are already widespread in many schools in Switzerland and other European countries. In contrast to other educational robotics systems, Thymio TPL does not have to be purchased as an additional accessory, hence considerably facilitating the access to a tangible interface. Another benefit of the devised platform is that it allows for a customization of the TPL blocks. The block symbols can thus be easily adapted to fit the needs of a class (e.g. simplified, non-parametric symbols for younger students, or more complex symbols to introduce the notions of variables and functions to more advanced students). This provides teachers the opportunity to specifically adjust the presented blocks based on the level of their students. In terms of adaptability, this feature represents another advantage of the devised platform. Students may even be asked to design and fabricate their own hand-drawn symbols, allowing for a more personalized learning experience. Such approaches may not only be more intuitive to novices, but they may also better harness the pedagogical concept of constructionism underlying educational robotics activities.

\section{CONCLUSION}

This work presented the development of a low-cost solution for a tangible programming language for Thymio, an educational robot widely used in schools in Switzerland and other European countries. User studies with 77 university students showed that the platform appeared to be more inviting to the participants than its screen-based graphical counterpart, encouraging the students to explore and discover its functionalities. The devised platform has shown to increase students' interaction both with the platform and with each other. Moreover, the use of simple craft materials for the fabrication of the physical blocks of the interface facilitates the reproduction of the platform by teachers and students and may therefore support the dissemination of the devised platform in formal education settings. In this context, the findings of this work may be considered a promising basis for further works in this direction.

\section{ACKNOWLEDGMENT}

The authors would like to thank all the students who participated in the studies. Moreover, they would like to thank Vaios Papaspyros and Yves Piguet for their support during the preparation and the execution of the studies.

\section{REFERENCES}

[1] D. Alimisis, "Educational robotics: Open questions and new challenges," Themes Sci. Technol. Educ., vol. 6, no. 1, pp. 63-71, 2013.

[2] F. B. V. Benitti, "Exploring the educational potential of robotics in schools: A systematic review," Comput. Educ., vol. 58, no. 3, pp. 978-988, 2012.

[3] S. Papavlasopoulou, M. N. Giannakos, and L. Jaccheri, "Reviewing the affordances of tangible programming languages: Implications for design and practice," IEEE Glob. Eng. Educ. Conf. EDUCON, no. April, pp. 1811-1816, 2017.

[4] P. Blikstein et al., "Project Bloks : designing a development platform for tangible programming for children," 2016.

[5] M. S. Horn, E. T. Solovey, R. J. Crouser, and R. J. K. Jacob, "Comparing the use of tangible and graphical programming languages for informal science education," Proc. 27th Int. Conf. Hum. factors Comput. Syst. CHI 09, vol. 32, p. 975, 2009.

[6] L. Xie, A. N. Antle, and N. Motamedi, "Are tangibles more fun? Comparing Children's Enjoyment and Engagement Using Physical, Graphical and Tangible User Interfaces," Proc. 2nd Int. Conf. Tangible Embed. Interact. - TEI '08, pp. 191-198, 2008.

[7] T. Sapounidis, S. Demetriadis, and I. Stamelos, "Evaluating children performance with graphical and tangible robot programming tools," Pers. Ubiquitous Comput., vol. 19, no. 1, pp. 225-237, 2015.

[8] K. M. Inkpen, W. Ho-Ching, O. Kuederle, S. D. Scott, and G. B. D. Shoemaker, "This is fun! we're all best friends and we're all playing: supporting children's synchronous collaboration," Int. Soc. Learn. Sci., p. 31, 1999.

[9] C. Giang, A. Piatti, and F. Mondada, "Heuristics for the Development and Evaluation of Educational Robotics Systems," IEEE Trans. Educ., pp. 1-10, 2019.

[10] M. Chevalier, F. Riedo, and F. Mondada, "Pedagogical Uses of Thymio II," IEEE Robot. Autom. Mag., vol. 23, no. 2, pp. 16-23, 2016.

[11] J. Shin, R. Siegwart, and S. Magnenat, "Visual Programming Language for Thymio II Robot," Interact. Des. Child., 2014.

(C) 2019 IEEE. Personal use of this material is permitted. Permission from IEEE must be obtained for all other uses, in any current or future media, including reprinting/republishing this material for advertising or promotional purposes, creating new collective works, for resale or redistribution to servers or lists, or reuse of any copyrighted component of this work in other works. 